\title{
Overcoming microbial hurdles: keeping the Olympics infection-free
}

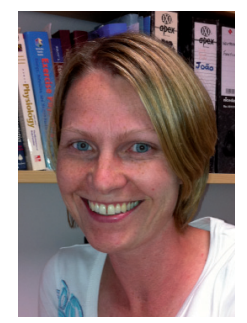

"... is it possible to keep the Olympics infection free? ... single bouts of prolonged strenuous exercise are associated with a temporary suppression of many aspects of immune function, and this may provide a 'window of opportunity' for infections to enter the body."

\section{Nicolette C Bishop}

School of Sport, Exercise \& Health Sciences, Loughborough University, Loughborough, Leicestershire, UK n- Tel.: +44 1509226385 n Fax: +44 1509226301 nn.c.bishop@lboro.ac.uk

In the coming weeks, over 11,000 athletes are expected to arrive in the UK for the XXX Olympic Games and XV Paralympic Games, or as it is more commonly known, London 2012. The arrival of so many athletes, coaches and other support staff from over 200 nations, many of which will be living in close proximity in the Olympic Village, provides an ideal environment for the transmission of infectious diseases. Of course, this situation is not uncommon for any large gathering, but for the athletes arriving in London over the next few weeks, this is the culmination of years of hard work, dedication and sacrifice for the chance to perform (and hopefully win) on the Olympic stage. Contracting an infection at any time can lead to absence from training and impaired performance. Contracting an infection during the Games can be devastating.

"The ability to withstand infections during periods of heighted physiological and psychological strain could actually be a prerequisite for elite performance."

Anecdotal reports from both athletes and coaches stretching back over 60 years document a high frequency of symptoms associated with respiratory infections in athletes. In support of this notion, there is now a large body of published literature suggesting that highly trained and elite athletes suffer more frequently from symptoms of upper respiratory infections (URIs) than those who are sedentary or who are recreationally active [1]. For example, 13\% of a cohort of over 2000 runners in the 1987 Los Angeles Marathon reported symptoms of URIs during the week after the event, compared with only $2 \%$ of a group of similarly experienced runners who did not compete. In addition, $40 \%$ of the runners experienced at least one episode of URI during the 2 months prior to the marathon itself [2]. Recent analysis of the 126 reported illnesses in athletes competing at last year's World Athletics Championships in Daegu, South Korea, revealed that $40 \%$ of illnesses affected the upper respiratory tract, with confirmed infection in approximately $20 \%$ of cases [3]. Other than sickness associated with exercise-induced dehydration (12\% of cases), gastroenteritis/diarrhea was the next most common illness reported (10\% of cases).

So is it possible to keep the Olympics infection free? It is now widely accepted that single bouts of prolonged strenuous exercise are associated with a temporary suppression of many aspects of immune function, and this may provide a 'window of opportunity' for infections to enter the body [1]. These effects may be cumulative over a period of time, so that during periods of heavy training and competition, athletes' immune systems may become chronically suppressed. Management of training load and recovery to maintain immune health is therefore crucial to minimize infection incidence in athletes. Having said this, there are several other factors that can also influence this risk. Poor dietary practices, heightened psychological stress, and poor sleep quality and quantity all impact on immune function and subsequent risk of infection [4]. Furthermore, practices such as sharing drinking bottles, a lack of hand washing, coming into contact with infected people, consuming contaminated food or beverages and living in close proximity to others can all increase the transmission of infectious pathogens. With this in mind, several practical lifestyle, nutritional and hygiene strategies to minimize infection risk in athletes

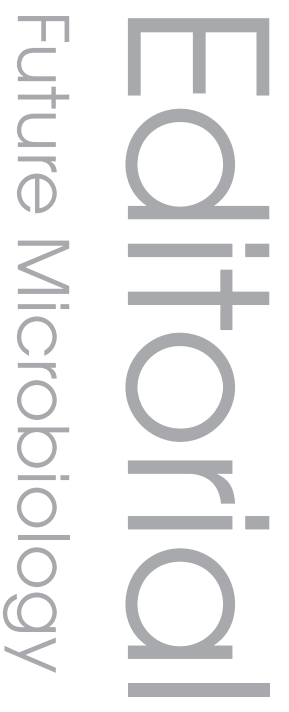

\section{Keywords}

- allergy = athletes = immunity

- upper respiratory infection

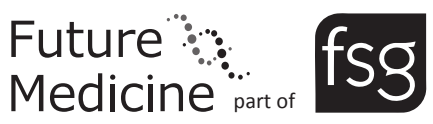


have been suggested, including avoiding people with infectious illness where possible, practicing good personal hygiene (washing hands, brushing teeth regularly), not sharing drinking bottles or cutlery, getting adequate sleep, managing psychological stress, maintaining energy and fluid balance, and considering vaccinations particularly against seasonal influenza. These should become part of an athlete's normal routine.

The ability to withstand infections during periods of heighted physiological and psychological strain could actually be a prerequisite for elite performance. In a recent study of URI symptoms, reported by 80 endurance-trained athletes over a 4-month winter period, we found that those athletes who were illness-prone had lower levels of salivary IgA secretion and higher release of IL-10 from antigen-stimulated blood cultures [5]. The identification of biomarkers for subsequent infection risk in athletes is certainly a key area of focus and future investigations will no doubt also look to identify possible genetic variations associated with an illness-prone phenotype. Identification of athletes who are naturally predisposed to infections would enable better management of infection risk to allow athletes to continue to train and perform optimally.

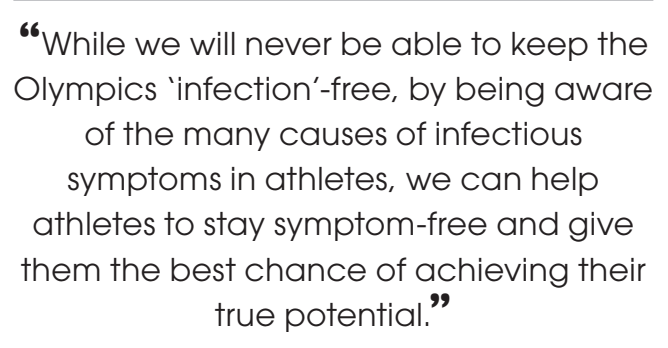

While it is certainly true that symptoms of respiratory illness are commonly reported in athletes, an infectious cause of these symptoms has not always been confirmed. A study examining the causes of symptoms of respiratory illness in both elite and recreational triathletes, and sedentary control subjects of similar age over a 5-month period found that only 11 out of 37 illness episodes had identifiable infectious cause [6]. Rhinovirus was the most commonly identified pathogen, with a small number of bacterial pathogens, including Streptococcus pyogenes, Haemophilus influenzae and Mycoplasma pneumoniae also responsible. This aside, almost $75 \%$ of reported symptoms of URI tested negative for detectable infectious agents. Even sports physicians can overestimate the occurrence of actual infections. In an evaluation study of symptoms of URI in elite Australian athletes, physicians characterized $89 \%$ of cases as viral or bacterial URIs, yet only $57 \%$ of cases were actually associated with an identified pathogen or laboratory measure consistent with infection [7].

In order to implement effective strategies to both prevent and manage respiratory illnesses in athletes, the cause of the symptoms needs to be clearly identified and this itself is an area of current debate. In the studies of Spence et al. [6] and Cox et al. [7], the fact that causes were unidentified does not necessarily mean that these illnesses were not infectious; there are many reasons why a pathogen may not be identified, including the limitations of current diagnostic techniques and existence of as yet unknown pathogens. Having said this, there is now a convincing line of thought that airway inflammation may be at least partly responsible for the illnesses without identifiable pathogenic cause. Lung ventilation rates can increase 10-20-fold with intensive exercise. This can damage the airways causing an increase in airway osmolarity and stimulating bronchial epithelial cells to release chemoattractants to promote the movement of immune cells into the airways [8]. The resulting noninfectious inflammation leads to many of the same symptoms as infectious respiratory illnesses. Furthermore, allergy and exposure to environmental factors such as cold dry air, pollutants and chlorine-related products can all trigger an inflammatory response and subsequent symptoms of URI. In support of this, a recently published study found a strong correlation between the prevalence of allergy and post-race reporting of symptoms of URI in over 200 runners who had taken part in the 2010 London Marathon [9]. This suggests that a high proportion of the symptoms may be allergic, rather than infectious in nature. Allergy is both treatable and manageable if correctly diagnosed, yet is often overlooked. In the evaluation study of symptoms of URI in elite Australian athletes, laboratory investigation identified allergy in almost $40 \%$ of the athletes presenting with URI, yet the sports physicians did not consider allergy as a possible cause of these symptoms for any of the episodes [7]. This is a substantial proportion of cases and certainly suggests that screening for common allergens would be a wise addition to the recommended strategies to help minimize infectious symptoms in elite athletes.

Current guidance to help athletes avoid illness, particularly of the upper respiratory tract, is focused on minimizing pathogen transmission and maintaining immune competence. This seems sensible if we assume that these symptoms all have an infectious cause. With 
several reports suggesting that a substantial proportion of URI symptoms experienced by athletes are of noninfectious inflammatory cause, strategies to prevent respiratory illness in athletes need to also look beyond avoidance of bacterial or viral pathogen transmission. Ongoing clarification of both the causes of symptoms of respiratory illness in athletes and those athletes who are most vulnerable to these symptoms is necessary to help identify the most appropriate treatment and management strategies. While we will never be able to keep the Olympics 'infection' free, by being aware of the many causes of infectious symptoms in athletes, we can help athletes to stay symptomfree and give them the best chance of achieving their true potential.

\footnotetext{
Financial \& competing interests disclosure

The author has no relevant affiliations or financial involvement with any organization or entity with a financial interest in or financial conflict with the subject matter or materials discussed in the manuscript. This includes employment, consultancies, honoraria, stock ownership or options, expert testimony, grants or patents received or pending, or royalties.

No writing assistance was utilized in the production of this manuscript.
}

\section{References}

1. Walsh, NP, Gleeson M, Shephard RJ et al. Position statement. Part one: immune function and exercise. Exerc. Immunol. Rev. 17, 6-63, (2011).

2. Nieman, DC, Johansen LM, Lee JW, Arabatzis K. Infectious episodes in runners before and after the Los Angeles Marathon. J. Sports Med. Phys. Fitness 30, 316-328 (1990).

3. Alonso JM, Edouard P, Fischetto G, Adams B, Depiesse F, Mountjoy M. Determination of future prevention strategies in elite track and field: analysis of Daegu 2011 IAAF
Championships injuries and illnesses surveillance. Br. J. Sports Med. 46(7), 505-514 (2012).

4. Walsh NP, Gleeson M, Pyne DB et al. Position Statement. Part two: maintaining immune health. Exerc. Immunol. Rev. 17, 64-103, (2011).

5. Gleeson M, Bishop NC, Oliveira M, McCauley T, Tauler P, Muhamad AS. Respiratory infection risk in athletes: association with antigen-stimulated IL-10 production and salivary IgA secretion. Scand. J. Med. Sci. Sports 22, 410-417, (2012).

6. Spence L, Brown WJ, Pyne DB et al. Incidence, etiology, and symptomatology of upper respiratory illness in elite athletes. Med. Sci. Sports Exerc. 39(4), 577-586, (2007).

7. Cox AJ, Gleeson M, Pyne DB, Callister R, Hopkins WG, Fricker PA. Clinical and laboratory evaluation of upper respiratory symptoms in elite athletes. Clin. J. Sport Med. 18, 438-445 (2008).

8. Bermon S. Airway inflammation and upper respiratory tract infection in athletes: is there a link? Exerc. Immunol. Rev. 13, 6-14 (2007).

9. Robson-Ansley P, Howatson G, Tallent J et al. Prevalence of allergy and upper respiratory tract symptoms in runners of the London Marathon. Med. Sci. Sports Exerc. 44(6), 999-1004 (2012). 